\title{
Comportamiento frente a la corrosión del material compuesto 2124/SiC
}

\author{
J.A. LÓPEZ-CABALLERO ${ }^{1 *}$, E. MARTÍN ${ }^{2}$, M.T. BAILE², A. FORN² Y J.M. BASTIDAS ${ }^{1}$ \\ ${ }^{1}$ CENIM-Centro Nacional de Investigaciones Metalúrgicas, CSIC, Avda. Gregorio del Amo 8, 28040 Madrid \\ ${ }^{2}$ Escuela Politécnica Superior de Ingeniería Vilanova i la Geltrú, UPC, \\ Avda. Víctor Balaguer s/n, 08800 Vilanova i la Geltrú, Barcelona
}

\begin{abstract}
Se realiza un estudio comparativo del comportamiento frente a la corrosión de la aleación de aluminio 2124 reforzada con partículas de carburo de silicio, obtenida por pulvimetalurgia y con tratamientos térmicos T4 y T6. Los resultados experimentales muestran que el tratamiento térmico T6 induce la formación de numerosos intermetálicos que reducen la resistencia a la corrosión. Las partículas de carburo de silicio no tienen un comportamiento catódico con respecto a la matriz de aluminio, sin embargo, disminuyen la resistencia a la corrosión, ya que generan zonas más activas en la interfase matriz/ refuerzo, debido a la acumulación de dislocaciones, tensiones residuales y a la precipitación de intermetálicos.
\end{abstract}

Palabras clave: Corrosión, Materiales Compuestos, Aluminio, Tratamientos Térmicos.

\section{Corrosion behaviour of $2124 / \mathrm{SiC}$ composite material}

A comparative study was performed on the corrosion behaviour of an aluminium matrix composite reinforced with silicon carbide particles, obtained by powder metallurgic. The 2124 / $\mathrm{SiC}$ material was heat treated using $\mathrm{T} 4$ and $\mathrm{T} 6$ procedures. The T6 heat treatment induced the formation of several intermetallics and reducing the corrosion resistance. The silicon carbide particles did not have a cathodic behaviour as compared with the aluminium matrix. However, these particles produced a diminution in the corrosion resistance due to the formation of more active zones in the matrix/reinforced interface. These ceramic particles caused intermetallic precipitation and deformation originating in the surrounding zones localized corrosion.

Keywords: Corrosion, Composites Materials, Aluminium, Heat Treatments.

\section{INTRODUCCIÓN}

Se conoce que materiales compuestos se desarrollaron para mejorar las propiedades mecánicas de las aleaciones ligeras, mediante la incorporación de refuerzos cerámicos (1).

$\mathrm{Al}$ principio, se utilizaron refuerzos continuos tales como fibras y filamentos, que proporcionaban materiales con elevadas propiedades mecánicas, pero tenían un elevado coste de producción y generaban problemas de fabricación. Así, las aplicaciones de estos materiales se reservaron, exclusivamente, a la industria militar y aeroespacial. Sin embargo, la aparición de los refuerzos discontinuos (dispersoides, partículas, laminillas y fibras cortas) permitió reducir costes, aumentar la producción y el tipo de utilidad, permitiendo aplicaciones civiles (2).

La introducción de una segunda fase produce alteraciones en la matriz metálica y, es de prever que tenga consecuencias en la resistencia a la corrosión (3). Los principales fallos por corrosión se pueden clasificar del siguiente modo: formación de pilas galvánicas entre el refuerzo y la matriz (4), corrosión localizada en la interfase matriz/refuerzo (5), fallos asociados al propio proceso de fabricación y a los tratamientos térmicos (6).

El material compuesto 2124/SiC tiene aplicación en la industria aeronáutica, por ejemplo, puede substituir a las aleaciones Ti6Al4V en algunos componentes, como es el caso de los soportes de las palas del helicóptero EC120 de Eurocopter (7). Sus propiedades mecánicas pueden mejorar mediante la aplicación de diferentes tratamientos térmicos
(8), que afectan al tamaño y distribución de los precipitados. Así mismo, la presencia de intermetálicos puede afectar al comportamiento frente a la corrosión (9).

Este trabajo tiene por objetivo analizar el comportamiento frente a la corrosión del material compuesto 2124 / SiC con dos tratamientos térmicos convencionales, $\mathrm{T} 4$ y $\mathrm{T} 6$, en presencia de una solución de cloruro de sodio $(\mathrm{NaCl})$ al 3,5\%.

\section{EXPERIMENTAL}

El material compuesto 2124/SiC fue obtenido por pulvimetalurgia. Tiene un $17 \%$ en volumen de partículas de $\mathrm{SiC}$, con un tamaño medio de $1,4 \mu \mathrm{m}$ y una relación longitud/ diámetro de 1,5. La matriz de aluminio presenta un tamaño de grano de $1 \mu \mathrm{m}$, próximo al de los polvos de aluminio utilizados en el proceso. La Tabla I muestra la composición química, determinada por espectroscopia óptica de emisión (OES), del material ensayado. La barra obtenida por pulvimetalurgia fue posteriormente forjada en matriz abierta a $505{ }^{\circ} \mathrm{C}$. Tras un proceso de puesta en solución a $495^{\circ} \mathrm{C}$ durante $6 \mathrm{~h}$ se procedió a un temple en agua de las muestras. Una parte de la barra se

TABLA I. COMPOSICIÓN QUÍMICA DE LA ALEACIÓN 2124 (\%PESO).

\begin{tabular}{|c|c|c|c|c|c|c|c|c|}
\hline $\mathrm{Si}$ & $\mathrm{Fe}$ & $\mathrm{Cu}$ & $\mathrm{Mn}$ & $\mathrm{Mg}$ & $\mathrm{Cr}$ & $\mathrm{Zn}$ & $\mathrm{Ti}$ & $\mathrm{Al}$ \\
\hline 0,03 & 0,07 & 4,08 & 0,58 & 1,38 & 0,003 & 0,003 & 0,001 & resto \\
\hline
\end{tabular}


envejeció a temperatura ambiente (2124/SiC T4), y la otra a $150{ }^{\circ} \mathrm{C}$ durante $6 \mathrm{~h}(2124 / \mathrm{SiC} \mathrm{T} 6)$.

Para la evaluación de la resistencia a la corrosión en disolucion de $\mathrm{NaCl}$ al 3,5\%, a temperatura ambiente, se utilizaron técnicas electroquímicas de impedancia (EIS), en disoluciones aireadas, y curvas de polarización en disoluciones aireadas y desaireadas.

Las probetas se embutieron en una resina epoxi, la conexión eléctrica se hizo mediante un cable de cobre plastificado y la superficie de ensayo se desbastó mecánicamente hasta el grado 600, seguida de un lijado manual hasta el grado 1200 y desengrase con etanol.

Las curvas de polarización se obtuvieron utilizando un potenciostato EG\&G PARC, modelo 273A, en la configuración de tres electrodos. Como contraelectrodo se utilizó una malla de platino con un área de $4 \times 9 \mathrm{~cm}^{2}$, el electrodo de referencia utilizado fue de calomelanos saturado (ECS) y el electrodo de trabajo fue la probeta objeto de estudio. Las curvas de polarización se llevaron a cabo después de 30 min de inmersión en la disolución aireada de $\mathrm{NaCl}$ al 3,5\%. Los ensayos se realizaron comenzado con un barrido catódico desde $-1,3 \mathrm{~V}_{\mathrm{ECS}}$ hasta el potencial de corrosión $\left(\mathrm{E}_{\text {corr }}\right.$ ), seguido de un barrido anódico hasta $-0,6 \mathrm{~V}_{\mathrm{ECS}}$ a diferentes velocidades de polarización (v): 0,05, 0,166, 5 y $25 \mathrm{mV} / \mathrm{s}$. Así mismo, se realizaron curvas de polarización en disoluciones desaireadas (pasando una corriente de nitrógeno $\left(\mathrm{N}_{2}\right)$, burbujeo, a través de la disolución durante $35 \mathrm{~min}$, antes de introducir el electrodo de trabajo, y manteniendo un flujo constante de $\mathrm{N}_{2}$ durante todo el ensayo para evitar la entrada de oxigeno) y siguiendo la misma metodología que con las disoluciones aireadas, a una velocidad de polarización de $0,166 \mathrm{mV} / \mathrm{s}$.

Las medidas de EIS se llevaron acabo utilizando un analizador de respuesta en frecuencia (FRA) de la marca Solartron, modelo 1250, conectado al potenciostato anterior, imponiendo una señal sinusoidal de amplitud $5 \mathrm{mV}$. Se obtuvieron diagramas de impedancia en el intervalo de frecuencia de $55 \mathrm{kHz}$ a $1,38 \mathrm{mHz}$ y con un barrido de frecuencia de 5 pasos/década. Las medidas de EIS se realizaron en el $\mathrm{E}_{\text {corr }}$
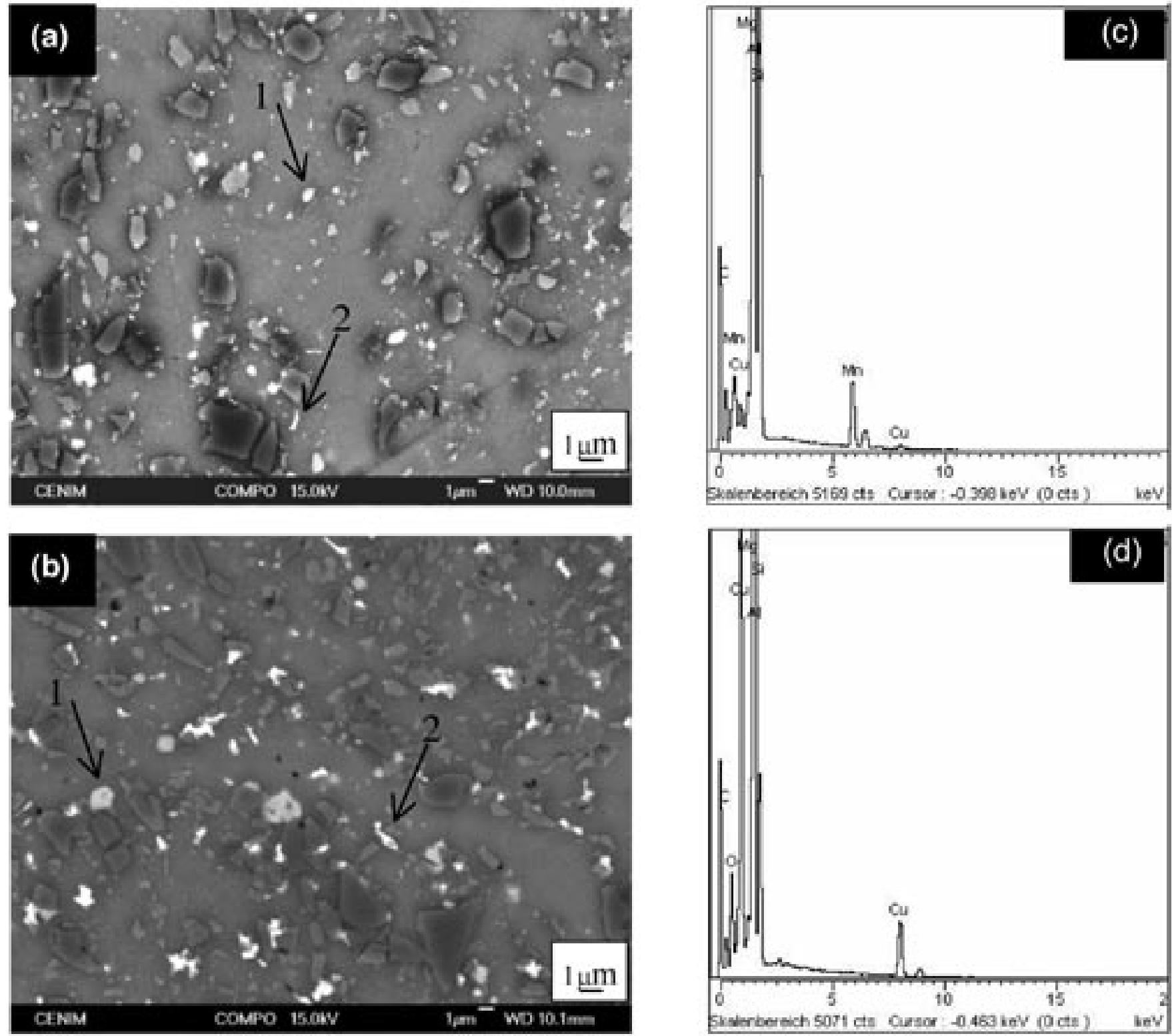

Fig. 1.- Micrografías MEB utilizando electrones retrodispersados, (a) 2124/SiC T4, (b) 2124/SiC T6 y espectros EDX, (c) intermetálico tipo 1 y (d) intermetálico tipo 2. 
después de 30 min de inmersión en una disolución aireada de $\mathrm{NaCl}$ al 3,5\%.

Para estudiar la morfología de las picaduras se polarizaron las probetas hasta $-0,3 \mathrm{~V}_{\mathrm{ECS}}$ en una disolución aireada de $\mathrm{NaCl}$ al 3,5\%, es decir, unos $300 \mathrm{mV}$ por encima del potencial de picadura $\left(\mathrm{E}_{\mathrm{pic}}\right)$. Posteriormente, al objeto de analizar la profundidad y extensión de las picaduras, se realizaron cortes transversales de las probetas y se pulieron utilizando sílice de $1 \mu \mathrm{m}$. El análisis micrográfico se realizó mediante un microscopio electrónico de barrido (MEB), JEOL JSM-6500-F, equipado con un analizador de energías dispersivas de rayos $\mathrm{x}$ (EDX), modelo Oxford Inca, y un microscópico óptico LEICA, modelo Q500Mw.

\section{RESULTADOS Y DISCUSIÓN}

La Fig. 1 muestra dos imágenes obtenidas mediante MEB, utilizando electrones retrodispersados, y dos espectros EDX de los intermetálicos de los materiales 2124/SiC T4 y 2124/SiC T6. En las micrografías puede observarse la matriz de aluminio (gris claro), las partículas de $\mathrm{SiC}$ (gris oscuro) y dos tipos de intermetálicos: los intermetálicos de forma redondeada, tipo 1, que contienen $\mathrm{Mn}-\mathrm{Cu}-\mathrm{Mg}-\mathrm{Al}$ y los de formas irregulares, tipo 2, que contienen $\mathrm{Cu}-\mathrm{Mg}-\mathrm{Al}$, probablemente $\mathrm{Al}_{2} \mathrm{Cu}$ (10-12). Los correspondientes análisis mediante EDX se muestran en la Fig. 1(c) y Fig. 1(d). En general, la distribución de las partículas de refuerzo es homogénea. No se observa la presencia de $\mathrm{Al}_{4} \mathrm{C}_{3}$ en la interfase refuerzo/matriz, debido a que durante el proceso de fabricación no se superaron $\operatorname{los} 710^{\circ} \mathrm{C}$, necesarios para su formación (13). Una de las principales características de este material es su baja porosidad (inferior al 0,1\%) debido al proceso de forja en caliente (7), los microporos son difíciles de detectar incluso mediante MEB. La gran cohesión entre las partículas de $\mathrm{SiC}$ y la matriz de aluminio es muy elevada y no se han detectado discontinuidades en la interfase matriz/ refuerzo. En la Fig. 1(b) se observa que en el material con tratamiento T6 los precipitados que contienen $\mathrm{Mn}-\mathrm{Cu}-\mathrm{Mg}-\mathrm{Al}$ presentan un tamaño ligeramente superior a los del material con tratamiento T4 (Fig. 1(a)), localizados, preferentemente, en las proximidades de las partículas cerámicas. Al ser el fenómeno de precipitación un proceso controlado por difusión en estado sólido, la mayor temperatura alcanzada por la puesta en solución durante el tratamiento T6 favorece la formación de precipitados, más o menos coherentes que son la causa del envejecimiento del material. La nucleación de segundas fases se ve favorecida en la interfase matriz/refuerzo, debido a la mayor densidad de dislocaciones generadas en estas interfases por efecto de las tensiones térmicas inducidas por el temple (14).

La Fig. 2 muestra las curvas de polarización obtenidas a las velocidades de polarización (v) de 0,166, 5 y $25 \mathrm{mV} / \mathrm{s}$. A medida que aumenta $v$ se produce un desplazamiento del Epic hacia valores más nobles. Así mismo, a medida que disminuye $v$ se favorece el control catódico del proceso de corrosión. En los ensayos a 0,166 mV/s, cuando se alcanza el $\mathrm{E}_{\text {corr }}$ se inicia un rápido aumento de la densidad de corriente, lo que pone de manifiesto que también se ha alcanzado el $\mathrm{E}_{\mathrm{pic}}$ (15). En todas las condiciones ensayadas, una vez superado el $\mathrm{E}_{\text {pic }}$ se observa la formación de burbujas de hidrógeno procedentes de la reducción de $\mathrm{H}^{+}$en la interfase aluminio/ electrolito $(16,17)$. En la Tabla II se observa que a las menores velocidades de polarización $(0,05$ y 0,166 $\mathrm{mV} / \mathrm{s})$ el $\mathrm{E}_{\text {pic }}$ presenta valores similares. Se determinó el $\mathrm{E}_{\text {pic }}$ a $0,166 \mathrm{mV} / \mathrm{s}$ realizando

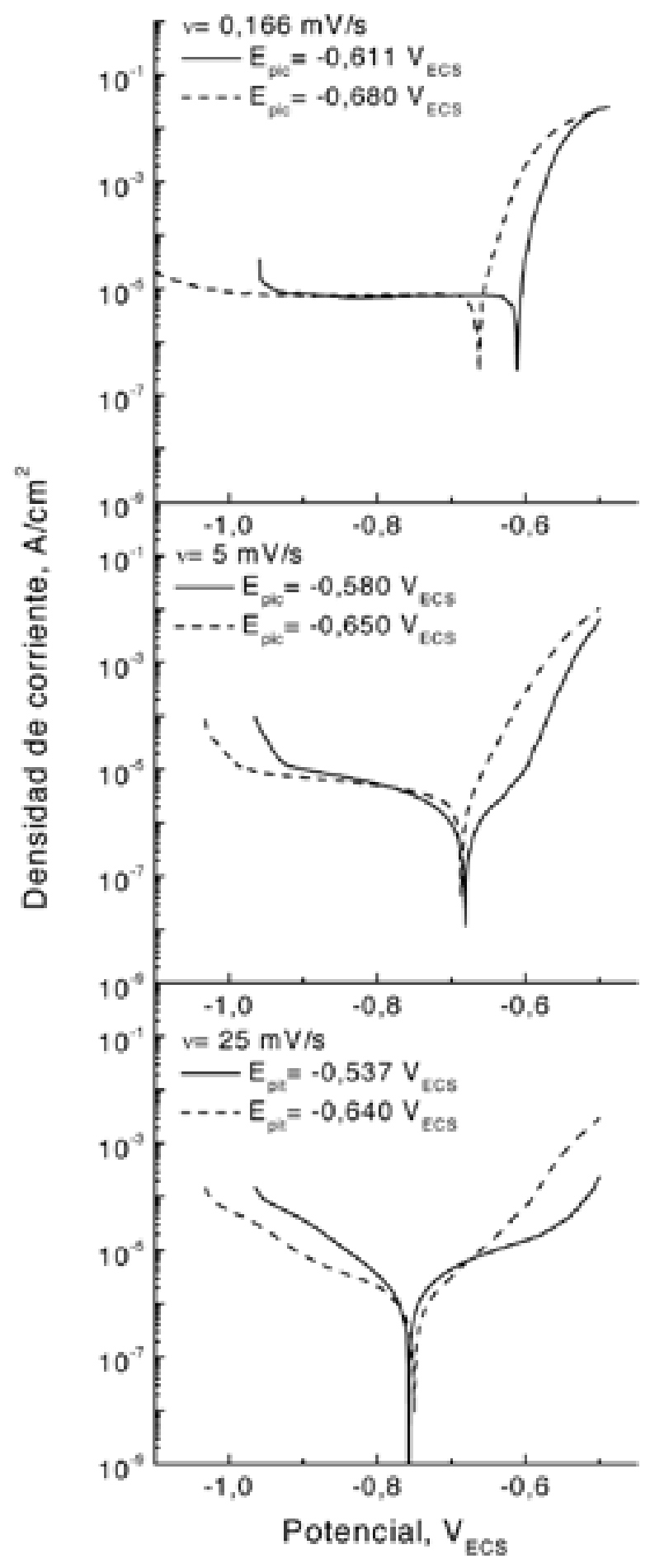

Fig. 2.- Curvas de polarización, (-) 2124/SiC T4 y (--) 2124/SiC T6, en disolución aireada de $\mathrm{NaCl}$ al 3,5\%.

TABLA II. POTENCIAL DE PICADURA ( $\mathrm{E}_{\mathrm{pic}}$ ) DE LOS MATERIALES 2124/SIC T4 Y 2124/ SIC T6 EN NaCl AL 3,5\%

\begin{tabular}{|c|c|c|}
\hline \multicolumn{3}{|c|}{$\mathrm{E}_{\mathrm{pic}^{\prime}} \mathrm{V}_{\mathrm{ECS}}$} \\
\hline $\mathrm{v}, \mathrm{mV} / \mathrm{s}$ & $2124 / \mathrm{SiC} \mathrm{T4}$ & $2124 / \mathrm{SiC} \mathrm{T} 6$ \\
\hline 0,05 & $-0,617$ & $-0,689$ \\
\hline 0,166 & $-0,611$ & $-0,680$ \\
\hline 5 & $-0,580$ & $-0,650$ \\
\hline 25 & $-0,537$ & $-0,640$ \\
\hline
\end{tabular}




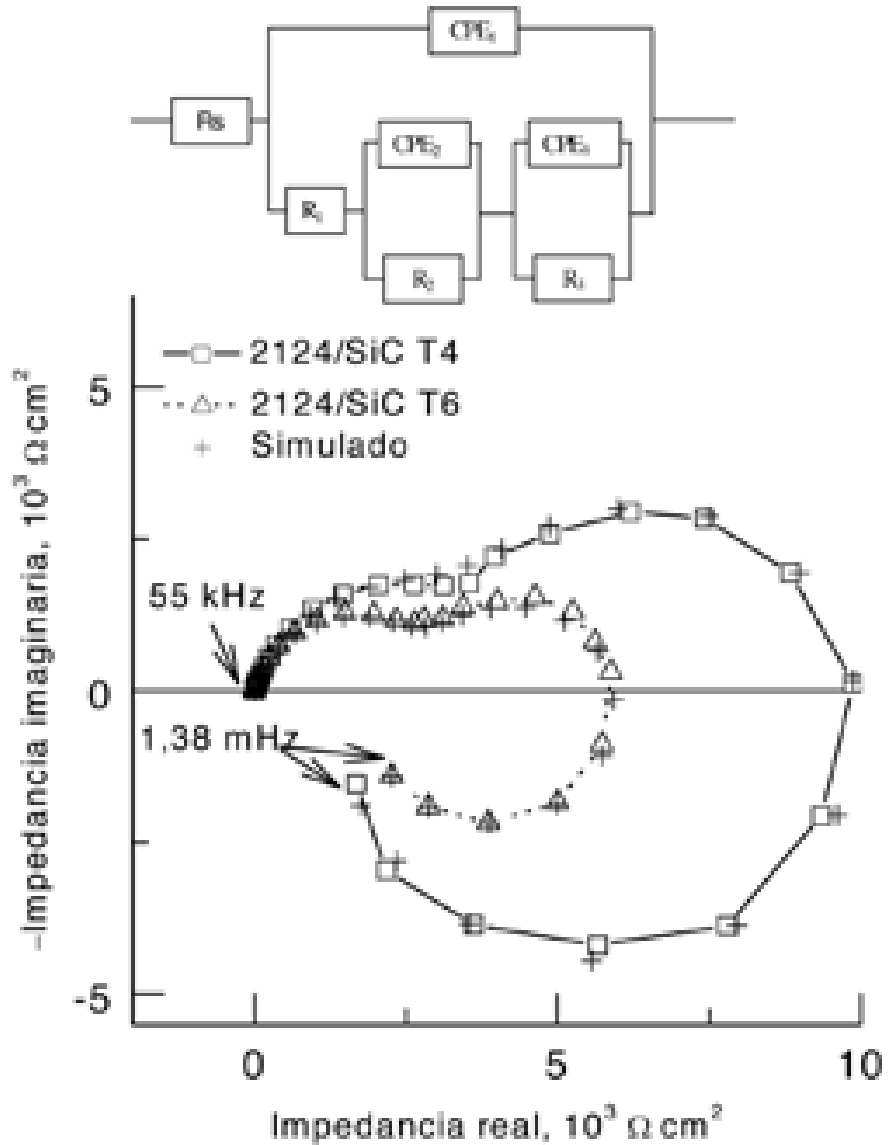

Fig. 3.- Diagramas de Nyquist, $(-\square-) 2124 / \operatorname{SiC} \mathrm{T} 4$ y $(\cdots \cdots \Delta \cdots \cdots) 2124$ / $\mathrm{SiC}$ T6, en disolución aireada de $\mathrm{NaCl}$ al 3,5\% y en el $\mathrm{E}_{\text {corr }}$.

curvas de polarización en disolución desaireada de $\mathrm{NaCl}$ al $3,5 \%$ (mediante burbujeo con $\mathrm{N}_{2}$ ) obteniéndose los siguientes valores: $-0,622 \mathrm{~V}_{\mathrm{ECS}} \mathrm{y}-0,682 \mathrm{~V}_{\mathrm{ECS}}$ para $2124 / \mathrm{SiC} \mathrm{T} 4$ y $2124 / \mathrm{SiC}$ T6, respectivamente. Estos valores se corresponden con los descritos en la Tabla II para condiciones aireadas, es decir el $\mathrm{E}_{\mathrm{pic}}$ no se ve afectado por la presencia de oxígeno (3).

Estos resultados confirman que el material con tratamiento $\mathrm{T} 4$ tiene un $\mathrm{E}_{\mathrm{pic}}$ más noble $\mathrm{y}$, en consecuencia, mayor resistencia a la corrosión que con tratamiento T6.

La Fig. 3 muestra dos diagramas de Nyquist obtenidos en el Ecorr y en disoluciones aireadas de $\mathrm{NaCl}$ al 3,5\%. A frecuencias elevadas e intermedias se observan dos semicírculos capacitivos, simulados por los subcircuitos CPE1$\mathrm{R} 1$ y $\mathrm{CPE}_{2}-\mathrm{R}_{2}$, respectivamente (ver circuito equivalente de la Fig. 3). A bajas frecuencias se observa un proceso inductivo, simulado por el subcircuito $\mathrm{CPE}_{3}-\mathrm{R}_{3}$. En la Tabla III se incluyen los valores de los parámetros utilizados en la simulación: Rs es la resistencia del electrolito; $R_{1}$ es la resistencia iónica dentro de las picaduras; $\mathrm{CPE}_{1}$ se asocia con la interfaseóxido/ electrolito; $R_{2}$ se relaciona con la resistencia del proceso de corrosión; $\mathrm{CPE}_{2}$ está relacionado con la interfase aluminio/
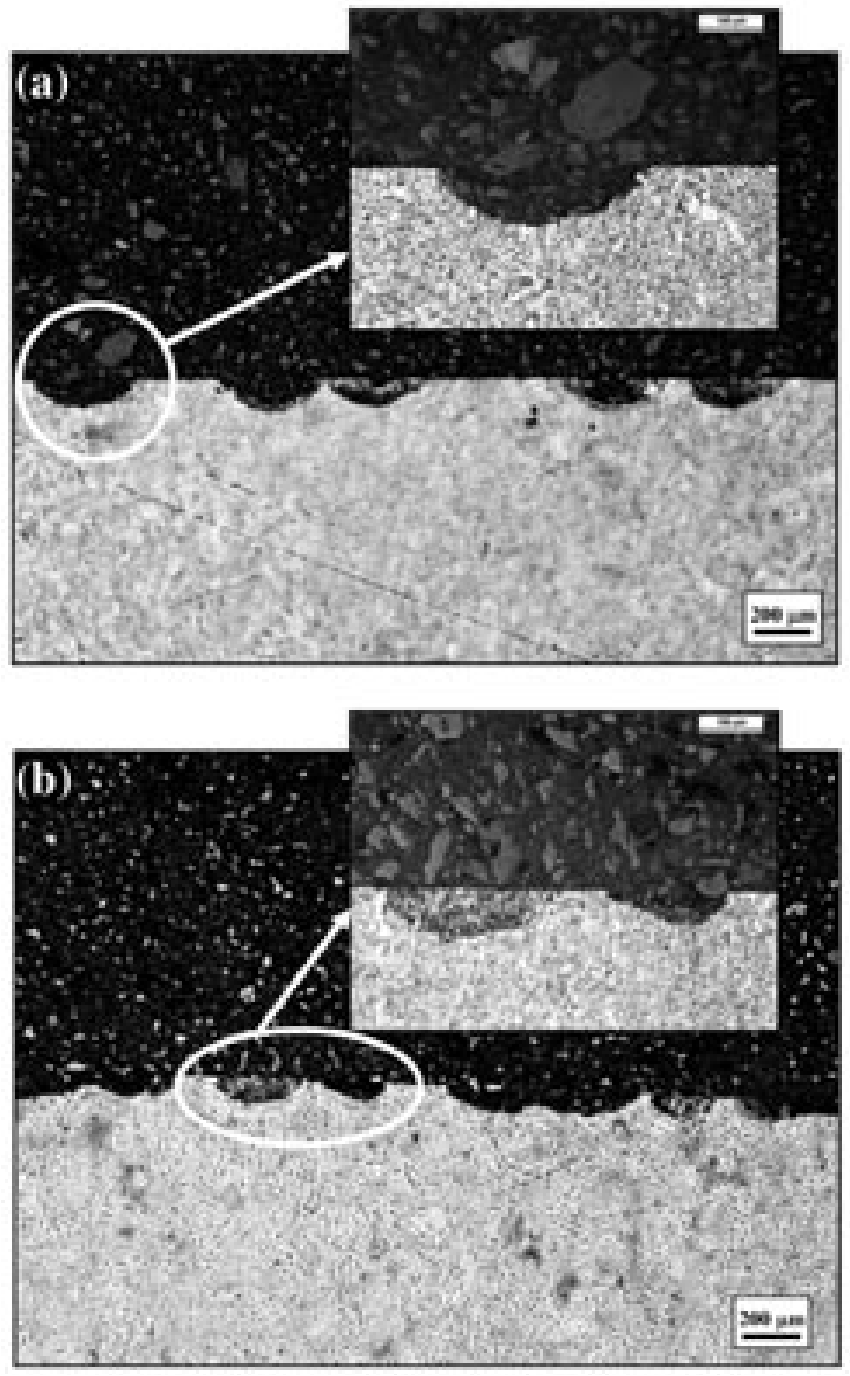

Fig. 4.- Micrografías ópticas de una sección transversal de la zona atacada, (a) 2124/SiC T4 y (b) 2124/SiC T6.

electrolito; $\mathrm{R}_{3}$ esta relacionado con la resistencia del proceso de adsorción; y $\mathrm{CPE}_{3}$ esta relacionado con el proceso de adsorción en la interfase aluminio/electrolito (18-20). Se observa una excelente concordancia entre los datos experimentales y los simulados.

En el circuito equivalente de la Fig. 3, se ha utilizado un CPE (constant phase element) para la simulación de los datos de impedancia. Un CPE es un elemento eléctrico distribuido, definido por la función empírica de admitancia: $Y=Y_{p(j \omega) \alpha^{\prime}}$ donde $\mathrm{Yp}$ es una constante, independiente de la frecuencia, con dimensiones $\Omega^{-1} \mathrm{~cm}^{-2} \mathrm{~s}^{\alpha} \mathrm{o}$, también, $\mathrm{F} / \mathrm{cm}^{2} \mathrm{~s}^{1-\alpha} ; \mathrm{j}^{2}=(-1) ; \omega$ es la frecuencia angular $(\omega=2 \pi \mathrm{f})(\mathrm{rad} / \mathrm{s}) ; \mathrm{y} \mathrm{f}$ es la frecuencia (Hz). El exponente adimensional $\alpha$ es: $-1 \leq \alpha \leq+1$. Si $\alpha=0$ el CPE representa una resistencia ideal, cuando $\alpha=+1$ el CPE es un condensador ideal, y cuando $\alpha=-1$ el CPE es una bobina (21).

TABLA III. PARÁMETROS UTILIZADOS EN LA SIMULACIÓN DE LOS DATOS DE IMPEDANCIA DE 2124/SiC T4 Y 2124/SiC T6 EN NaCl AL 3,5\%

\begin{tabular}{|c|c|c|c|c|c|c|c|c|c|c|}
\hline Material & $\begin{array}{c}\mathrm{R}_{\mathrm{s}^{\prime}} \\
\Omega \mathrm{cm}^{2}\end{array}$ & $\begin{array}{c}\mathrm{Y}_{\mathrm{P1}^{\prime}} \\
\mu \mathrm{F} / \mathrm{cm}^{2} \mathrm{~s}^{1-\alpha 1}\end{array}$ & $\alpha_{1}$ & $\begin{array}{c}\mathrm{R}_{1^{\prime}} \\
10^{3} \Omega^{\mathrm{cm}^{2}}\end{array}$ & $\begin{array}{c}\mathrm{Y}_{\mathrm{P} 2} \\
\mu \mathrm{F} / \mathrm{cm}^{2} \mathrm{~s}^{1-\alpha 2}\end{array}$ & $\alpha_{2}$ & $\begin{array}{c}\mathrm{R}_{2^{\prime}} \\
10^{3} \Omega^{2} \mathrm{~cm}^{2}\end{array}$ & $\begin{array}{c}\mathrm{Y}_{\mathrm{P}{ }^{\prime}} \\
\mu \mathrm{F} / \mathrm{cm}^{2} \mathrm{~s}^{1-\alpha 3}\end{array}$ & $\alpha_{3}$ & $\begin{array}{c}\mathrm{R}_{3^{\prime}} \\
10^{3} \Omega^{\mathrm{cm}}{ }^{2}\end{array}$ \\
\hline $\begin{array}{l}2124 / \mathrm{SiC} \mathrm{T} 4 \\
2124 / \mathrm{SiC} \text { T6 }\end{array}$ & $\begin{array}{l}5 \\
5\end{array}$ & $\begin{array}{l}40 \\
37\end{array}$ & $\begin{array}{l}0,805 \\
0,841\end{array}$ & $\begin{array}{l}5 \\
3\end{array}$ & $\begin{array}{l}310 \\
743\end{array}$ & $\begin{array}{l}0,898 \\
0,830\end{array}$ & $\begin{array}{l}8 \\
4\end{array}$ & $\begin{array}{l}-1893 \\
-7198\end{array}$ & $\begin{array}{l}0,999 \\
0,994\end{array}$ & $\begin{array}{l}-11 \\
-5\end{array}$ \\
\hline
\end{tabular}



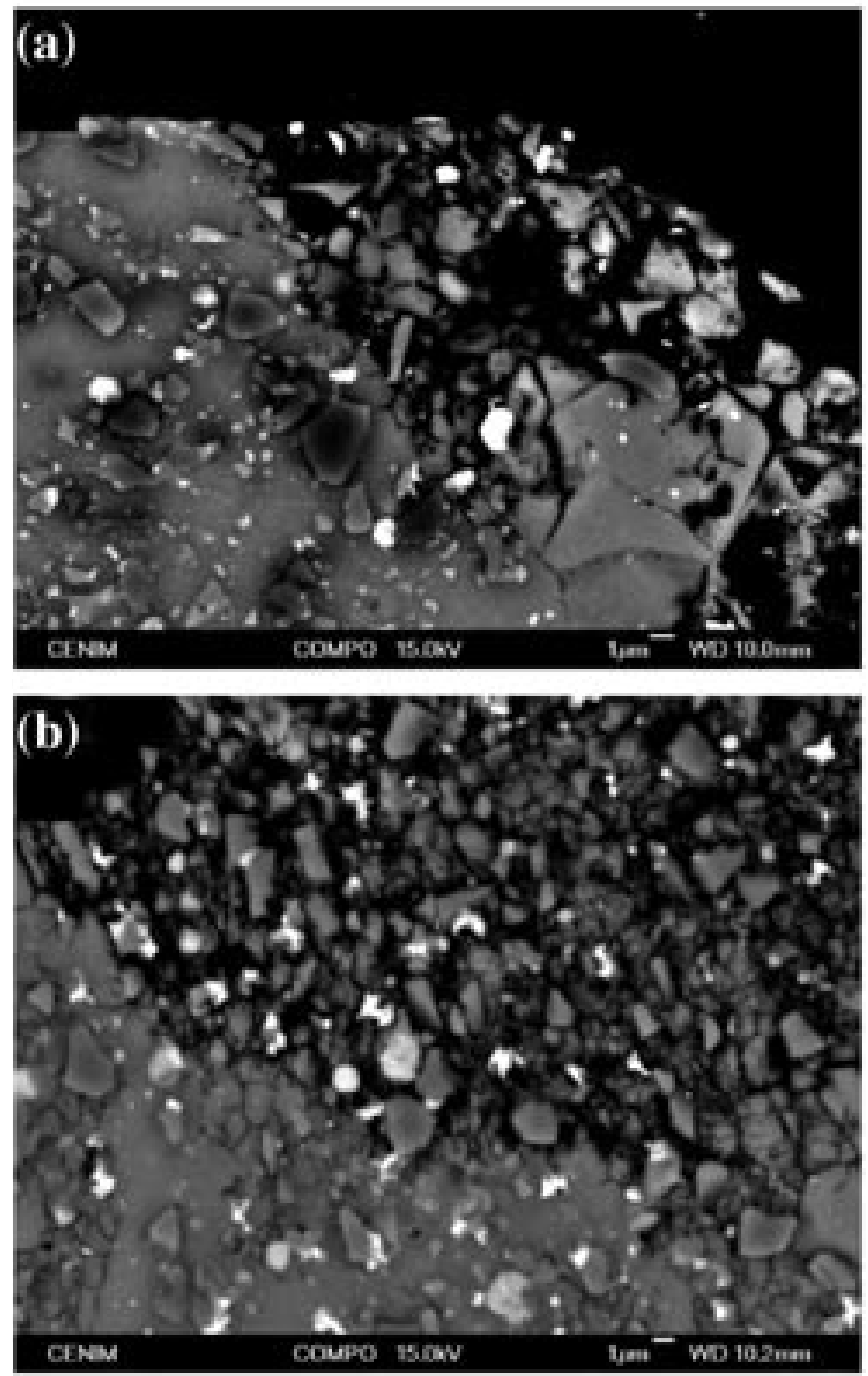

Fig. 5.- Micrografías MEB utilizando electrones retrodispersados del corte transversal de una picadura, (a) 2124/SiC T4 y (b) 2124/SiC T6.

Los resultados de la Tabla III muestran que el material con tratamiento $\mathrm{T} 4$ presenta un valor de $\mathrm{R}_{2}$ mayor que el material con tratamiento $\mathrm{T} 6$, lo que induce a pensar que el tratamiento térmico T4 presenta una mayor resistencia a la corrosión. El aumento de $Y_{\mathrm{p} 2}$ con el tratamiento térmico T6 es también indicativo de un proceso de corrosión más intenso (22), y de un aumento del tamaño o del número de precipitados intermetálicos (23).

La Fig. 4 muestra dos micrografías ópticas de secciones perpendiculares a la superficie atacada de los materiales 2124/SiC T4 y 2124/SiC T6 polarizados anódicamente hasta $-0,3 \mathrm{~V}_{\mathrm{EC}}$. En dicha figura se observa el grado de ataque y la profundidad de las picaduras. La máxima profundidad de picadura observada en el material $2124 / \mathrm{SiC}$ T4 es de $140 \mu \mathrm{m}$. En el material 2124/SiC T6 la medida de la profundidad de las picaduras fue menos precisa debido al elevado grado de deterioro superficial, aunque, tentativamente puede estimarse entorno a los $180 \mu \mathrm{m}$. El diámetro medio de las picaduras en el $2124 / \mathrm{SiC} \mathrm{T} 4$ es de $350 \mu \mathrm{m}$ y en el $2124 / \mathrm{SiC}$ T6 de 320 $\mu \mathrm{m}$. La profundidad y el diámetro de las picaduras en estos materiales reforzados es ligeramente mayor que en las mismas aleaciones sin reforzar (10).

Las picaduras que se observan en la Fig. 4, con forma semiesférica y distribuidas homogéneamente sobre toda la superficie, tienen un aspecto semejante al observado en los procesos de corrosión de la fase $\alpha$ en las aleaciones AlSi. En estas aleaciones las picaduras se han justificado por la formación de pilas de aireación diferencial, debido a la activación y pasivación alternativa de las superficies anódicas y catódicas (22).

La Fig. 5 muestra dos picaduras de las aleaciones reforzadas con tratamientos $\mathrm{T} 4$ y $\mathrm{T} 6$, obtenidas utilizando electrones retrodispersados. En ambos casos la forma de ataque es similar, aunque es mayor en el 2124 / SiC T6. Los precipitados de color claro corresponden a los identificados en la Fig. 1 y tienen carácter catódico respecto a la matriz. El ataque avanza a través de los límites de grano de la fase $\alpha$ y puede verse favorecido en la interfase matriz/refuerzo por la presencia de dislocaciones, precipitados intermetálicos $\mathrm{y} / \mathrm{o}$ tensiones residuales (10-12). La precipitación de intermetálicos, no detectados mediante MEB, podría facilitar el avance de las picaduras (24).

\section{CONCLUSIONES}

Los materiales compuestos 2124/SiC T4 y 2124/SiC T6 presentan una microestructura similar. Los precipitados intermetálicos se localizan, preferentemente, en las proximidades de las partículas de SiC.

El material con tratamiento T4 presenta un potencial de picadura más noble en las distintas velocidades de polarización ensayadas, tanto en disolución aireada como desaireada. A bajas velocidades de polarización el proceso de corrosión tiene control catódico.

Los diagramas de Nyquist muestran que el material con tratamiento T6 presenta menor resistencia a la corrosión que con tratamiento T4. Con T6 el valor de $Y_{p 2}$ es elevado, lo que es indicativo de una activación de la matriz de aluminio probablemente por una mayor proporción de intermetálicos. En ambos materiales, se observa un proceso inductivo a bajas frecuencias, posiblemente atribuible a un proceso de adsorción sobre la superficie metálica.

La forma de las picaduras es similar en los dos materiales ensayados, pero en el material con tratamiento térmico T6 hay mayor número de picaduras que en el material con tratamiento térmico T4 y se observa una mayor degradación superficial. En todos los casos el diámetro de las picaduras es mayor que el grado de penetración. El mecanismo de avance se produce, inicialmente, por los granos de fase $\alpha$, alrededor de las partículas de $\mathrm{SiC}$ y de los precipitados intermetálicos.

\section{AGRADECIMIENTOS}

Los autores desean expresar su agradecimiento a la Comisión Interministerial de Ciencia y Tecnología (CICYT) por la financiación del proyecto MAT2001-1732-C02 y a la Unión Europea por el proyecto BRITE-EURAM no BE96-3168.

\section{BIBLIOGRAFÍA}

1. M. M. Schwartz, «Joining Composite Matrix Materials», pp. 1-4, American Society for Metals International, Ohio, (1994).

2. S. M. Lee, «Handbook of Composites Reinforcements», pp. 350-357, VCH, California, (1993).

3. A. Turnbull, «Review of corrosion studies on aluminium metal matrix composites», Brit. Corros. J., 27 27-35 (1992). 
4. L. H. Hihara, R. M. Latanision, "Galvanic corrosion of aluminum-matrix composites», Corrosion, 48 546-552 (1992).

5. S. Payan, Y. Le Petitcorps, J. M. Olive, H. Saadaoui, «Experimental procedure to analyse the corrosion mechanisms at the carbon/aluminium interface in composite materials», Composites Part A, 32 585-589 (2001).

6. R. C. Paciej, V. S. Agarwala, «Metallurgical variables influencing the corrosion susceptibility of a powder metallurgy $\mathrm{SiCw} / \mathrm{Al}$ composite», Corrosion, 42 718-729 (1986).

7. Proyecto BRITE-EURAM no BE96-3168

8. E. Martín, A. Forn, R. Nogué, «Strain hardening behaviour and temperature effect on Al-2124/SiCp», J Mater. Proc. Tech., 143-144C 1-4 (2003).

9. J. F. McIntyre, R. K. Conrad, S. L. Golledge, «The effect of heat treatment on the pitting behaviour of SiCw/ AA2124», Corrosion, 46 902-905 (1990).

10. A. Forn, E. Martin, M. T. Baile, J. M. Bastidas, "Corrosion behaviour of AA2124 and AA359 reinforced with $\mathrm{SiC}$ particles», 15th International Corrosion Congress, Granada, (2002).

11. G. E. Kiourtsidis, S. M. Skolianos, «Corrosion behavior of squeeze-cast silicon carbide-2024 composites in aerated 3.5 wt. \% sodium chloride», Mater. Sci. Eng. A, 248 165-172 (1998).

12. G. E. Kiourtsidis, S. M. Skolianos, E. G. Pavlidou, «A study on pitting behaviour of AA2024/SiCp composites using the double cycle polarization technique», Corros. Sci., 41 1185-1203 (1999).

13. S. Li, R. J. Arsenault, P. Jena, «Bonding of $\mathrm{SiC}$ and Aluminium», pp. 33-37, Cast Reinforced Metal Composites Metal Conference, Chicago, (1988).

14. G. E. Kiourtsidis, S. M. Skolianos, G. A. Litsardakis, «Aging response of aluminium alloy 2024/ silicon carbide particles ( $\mathrm{SiCp}$ ) composites», Mater. Sci. Eng. A, 382 351-361 (2004).

15. J. M. Bastidas, J. L. Polo, C. L. Torres, E. Cano, «A stochastic approach to study localized corrosion of AISI 304L and 316L stainless steel as function of potential scan rate», Corrosion, 57 666-669 (2001).
16. D. M. Dražic, J. P. Popic, «Corrosion rates and negative differences effects for Al and some Al alloys», J. Appl. Electrochem., 29 43-50 (1999).

17. K. Sasaki, H. S. Isaacs, «Origins of electrochemical noise during pitting corrosion of aluminium», J. Electrochem. Soc., 151 B124-B133 (2004).

18. J. Hitzig, K. Jüttner, W. J. Lorenz, «AC-impedance measurements on corroded porous aluminium oxide films», J. Electrochem. Soc., 133 887-892 (1986).

19. J. M. Bastidas, J. L. Polo, C. L. Torres, E. Cano, "A study on the stability of AISI 316L stainless steel pitting corrosion through its transfer function», Corros. Sci., 43 269-281 (2001).

20. E. McCafferty, «Sequence of steps in the pitting of aluminium by chloride ions», Corros. Sci., 45 1421-1438 (2003).

21. J. L. Polo, E. Cano, N. Mora, D. Y. Kong, J. M. Bastidas, «Estudio comparativo de impedancia de la corrosión localizada de los aceros inoxidables 254 SMO y AISI 316L en soluciones que contienen cloruros», Bol. Soc. Esp. Ceram. V, (en prensa).

22. J. M. Bastidas, A. Forn, M. T. Baile, J. L. Polo, C. L. Torres, «Pitting corrosion of A357 aluminium alloy obtained by MEBisolid processing», Mater. Corros., 52 691- 696 (2001).

23. A. J. Griffin Jr, F. R. Brotzen, C. F. Dunn, «Impedance-spectroscopy response of aluminum copper silicon alloys», J. Electrochem. Soc., 141 3473-3479 (1994).

24. A. Ureña, J.M. Gomez-Salazar, L. Gil, M.D. Escalera, J.L. Baldonedo, «Scanning and transmission electron microscopy study of the microstructural changes occurring in aluminium matrix composites reinforced with $\mathrm{SiC}$ particles during casting and welding: interface reactions» J. Microsc-Oxford, 196124 (1999)

Recibido: 17.01.05

Aceptado: 25.08.05 